\title{
CANCER STEM CELLS - NEW APPROACH TO CANCEROGENENSIS AND TREATMENT
}

\author{
Zuzana Mačingová, Stanislav Filip \\ Charles University in Prague, Faculty of Medicine and University Hospital Hradec Králové, Czech Republic: \\ Department of Oncology and Radiotherapy
}

\begin{abstract}
Summary: Recently, there is an increasing evidence supporting the theory of cancer stem cells not only in leukemia but also in solid cancer. To date, the existence of cancer stem cells has been proven in acute and chronic myeloid leukemia, in breast cancer, in brain tumors, in lung cancer and gastrointestinal tumors. This review is focusing on the recent discovery of stem cells in leukemia, human brain tumors and breast cancer.

A small population of cells in the tumor (less than $1 \%$ ) shows the potential to give rise to the tumor and its growth. These cells have a substantial characteristic of stem cells - ability for self-renewal without loss of proliferation capacity with each cell division. Furthermore they are immortal, rather resistant to treatment and express typical markers of stem cells. The origin of these resident cancer stem cells is not clear. Whether the cancer stem cells originate from normal stem cells in consequence of genetic and epigenetic changes and/or redifferentiation from somatic tumor cells to the stem-like cells remains to be investigated. We propose the idea of the relation between normal tissue stem cells and cancer stem cells and their populations - progenitor cells. Based on this we highlight one of the major characteristic of stem cell - plasticity, which is equally important in the physiological regeneration process as well as carcinogenesis. Furthermore, we consider the microenvironment as a limiting factor for tumor genesis in AML, breast cancer and brain tumors.

Thus the biological properties of cancer stem cells are just beginning to be revealed, the continuation of these studies should lead to the development of cancer stem cells target therapies for cancer treatment.
\end{abstract}

Key words: Stem cells; Tumor stem cells; Carcinogenesis; Stem cell therapy

\section{Introduction}

Stem cells are characterized by their capability for unlimited proliferation and their ability to self-renew by symmetric or asymmetric division. Symmetric division is the process by which stem cells increase their absolute number. In the case of asymmetric division, the stem cell divide to generate a different, more specialized daughter cell with a limited capacity for both division and/or survival. Also the second daughter cell still must remain "stem" to generate cells for growth and to substitute for the loss of cells. Another defining feature of a stem cell is to differentiate into a variety of cell types.

The association between stem cells and cancer stem cells can be viewed from three aspects:

1) the similarities and differences in control and regulation of their capacity for self-renewal,

2) whether the normal tissue stem cells gives rise to the cancer cells,

3) whether cancer stem cells actually exist.

The mechanisms involved in their unique property of unlimited capacity to self-renew are not well described. How- ever they may be similar to mechanisms used in normal stem cells, for example the activity of telomerase's, apoptosis inhibitors and signal transduction pathways. Deregulated signaling pathways like Notch, Sonic hedgehog ( $\mathrm{SHH}$, hedgehog) and Wnt play a significant role in carcinogenesis.

The suggestion of cancer stem cells is more plausible simply due to the fact it is a farless complicated to change to the capacity for self-renewal than it would be reactivate an immortal program in an already differentiated cell. Furthermore, stem cells live longer than differentiated cells, so they are exposed to external factors for a much longer time. The accumulation of genetic defects/mutations can also cause misregulation.

Next we question the capacity for self-renewal of all tumor cells, and whether this is only due to the stem cancer cells or the presence of any other cells within the tumor tissue that might have the ability to generate a tumor. The concept that a rare population of tissue may be the cellular origin of cancer was proposed in $19^{\text {th }}$ century by pathologists such as Cohnheim and Durante. These scientists observed similarities between embryonic and cancer tissue 
with respect to their enormous capacity for proliferation and differentiation. Their observation led to the hypothesis that resting embryonic stem cells may reside in adult tissue and that upon activation these stem cells may acquire the ability to give rise to cancer (9). In the late 1930s scientists showed that leukemia can be transmitted from one mouse to another by using a single undifferentiated leukemia cell (38). Approximately 30 years later, using in-vitro colony formation assays, it was established that a rare subpopulation of acute myeloid leukemia (AML) possessed the ability of self-renewal and proliferation, and therefore going on to give rise to new tumors $(27,35,39)$. In the 1970 s researchers revisited this idea. The hypothesis about the stem cell potential of leukemia cells (AML) was formally tested in-vivo on a NOD/SCID mouse model (5). Studies that followed on from this had confirmed that there were similarities in the biology of stem cells and cancer stem cells. They proposed the idea that cancers may rise from quiescent tissue stem cells (32).

In 2001 studies were published with relevance to the steps that lead to carcinogenesis. These studies showed that the signal transduction pathways (such as Wnt and Hedgehog) play an important role in tumor genesis and how essential it is to consider the existence of stem cells and cancer stem cells regarding their roles in carcinogenesis $(32,45)$.

There are four main classes of stem cells:

1) Totipotent cells (after fertilization, created zygote is a totipotent cell, meaning it has the genetic potential to create every cell within the body, the nourishing placenta and extra-embryonic tissues).

2) Pluripotent cells (almost all types of blastocyst cells).

3) Multipotent cells (can give rise to other types of cells but it is limited in its ability to differentiate).

4) Unipotent cells (have a very limited ability to differentiate).

The association between cancer and stem cells and cancer was then further discussed for several decades (14, 46) and it was found that stem cells and cancer stem cells have similar features concerning: unlimited proliferation, capacity for tissue specific differentiation, clonogenic potential, the capacity of self-renew and the specific differentiation. Howecer differentiation is characteristic only for a specific cancer cell population; in some cancer cells terminal differentiation is defined, caused for example by loss of ability to proliferate, set of by external factors such as irradiation, chemical drugs.

The finding that stem cells isolated from fetal tissue are able to differentiate into a variety of cell types was a very important step in proving the plasticity of somatic cells or stem cells that are present within differentiated tissues (13). It also led to the theory of transdifferentiation. Simplified, it is the production of cells within tissues where they would not originally occur $(13,31)$. It was this knowledge that lead to the possible association between the plasticity of stem cells and carcinogenesis $(14,15,32)$. Plasticity of stem cells and cancer cells is based on the ability for self-renewal, this ability is very important, but also very dangerous.

\section{Cancer stem cells}

Recently, there have been two important issues raised directly related to both stem and cancer stem cells. Firstly, whether there is a relation between resident stem cells and cancer stem cells leading to tumor formation. Furthermore, whether there is a "minimal" number of these cells that act as a limiting factor. Secondly, whether the resident stem cells are results of transforming events that lead to the generation of tumor stem cells or from a different cell population that is secondarly transformed. For example, from a migrating population of stem cells after humoral stimulation in a new environment.

The subpopulation of cancer stem cells is responsible for tumor rise, growth and metastasis. It is believed that this happens because cancer stem cells have predetermined properties such as longevity and the self-renewal ability, similar to normal tissue stem cells. Once again a question is raised, what kind of relation is there between normal and cancer stem cells?

The ability of self-renewal is one of the limiting carcinogenesis factors. This was acknowledged in studies performed by a couple of laboratories. They identified small cell populations that were responsible for tumor formation and their experiments clearly showed the existence of cancer stem cells for leukemia, brain tumors and breast tumors (26).

Sell and Pierce, as proposers of the stem cell origin of cancer, originally suggested that malignancy leads to blocked differentiation and that the stem cells are targets of "firsthit" mutation. But stem cells, as a long-lived cell, are more likely to be the subject of mutations and furthermore cancer stem cells may exist in a quiescence state for a long time, keeping their ability to self-renew and therefor leading to the possible formation of cancer (37). The concept that stem cells are the cellular origin of cancer is presently accepted, but there many questions related to this model $(4,14)$.

\section{Hematopoietic cancer stem cells}

The first steps in proving the capability of hematopoietic stem cells in initiating the formation of acute myeloid leukemia (AML) were shown in experiments taken by Dick et al. It was demonstrated that in AML, only a small subpopulation of leukemic cells are capable of initiating leukemia in SCDI mice (5). Alternatively, Weissman et al supplemented the model by using hematopoitec progenitor cells - CMP (common myeloid progenitors), GMP (granulocytic/monocytic-restricted progenitors) and MEP (megakaryocytic/erythroid-restricted progenitors), as well as the role of some oncogenes and oncoproteins in the process of AML genesis (7) (Fig. 1). These studies employed the re- 
lation between MLL-ENL (mixed lineage leukemia-eleven nineteen leukemia) and its protein production and next his ability of up-regulation oncoprotein c-myc (36). MLL oncoproteins are constitutive activators of Hox genes (homeobox) and promote myeloid transformation (3). It is necessary to highlight that translocation between MLL gene an ANL gene $-\mathrm{t}(11,9)$ is linked to human AML (2). Bmi-1 also plays an important role in the self-renewal of normal HSCs as well as leukemic cells. A potential mechanism by which Bmi-1 regulates stem cell-renewal may be related to the regulation of survival and proliferation genes such as p19Arf, p $16^{\text {lnk4a }}$ and a p53 target gene, $\operatorname{Wigl}(21,29)$. Latest experimental findings refer to humoral factors and their ability to affect the transformation of normal stem cells at certain points in the cell cycle and to form cancer stem cells and finally to give rise to tumor.

\section{Breast cancer stem cells}

The existence of normal mammary stem cells was established as early as 1959, when DeOme and colleagues observed that cells extracted from epithelium isolated from several different regions of mammary gland were able to generate fully functional mammary outgrowths containing ductal, lobuloalveolar and myoepithelial cells (8). In order to identify and characterize these cells within the mammary gland several studies were performed. Based on them it has been concluded that the entire mammary gland can develop from a multipotent stem cell clone positioned throughout the gland (20). Furthermore, side population cells have been identified in the mammary gland and it has been demonstrated they can give rise to mammary ducts and alveoli (47). Side population cells expressing eg. SCA- $1^{+}$(stem cell antigen-1), $\mathrm{ESA}^{+}$(epithelial-specific antigen)/MUC-1 (sialomucin) are characterized by specific phenotypic and genotypic profile and misregulation of their signal transduction pathway. All of these characteristics predispose them to cancer stem cells genesis and consequently to give rise to progenitor cells. Clark et all was the first to identify this population of cells forming the breast cancer. These cells were defined as $\mathrm{CD} 44^{+} \mathrm{CD} 24^{-/ \text {low }}$. Cells with this specific cell-surface antigen transplanted into SCDI mice (immunodeficient mice) had a 10 - to 50 -fold increased ability to form tumors compared with the original tumor mass.

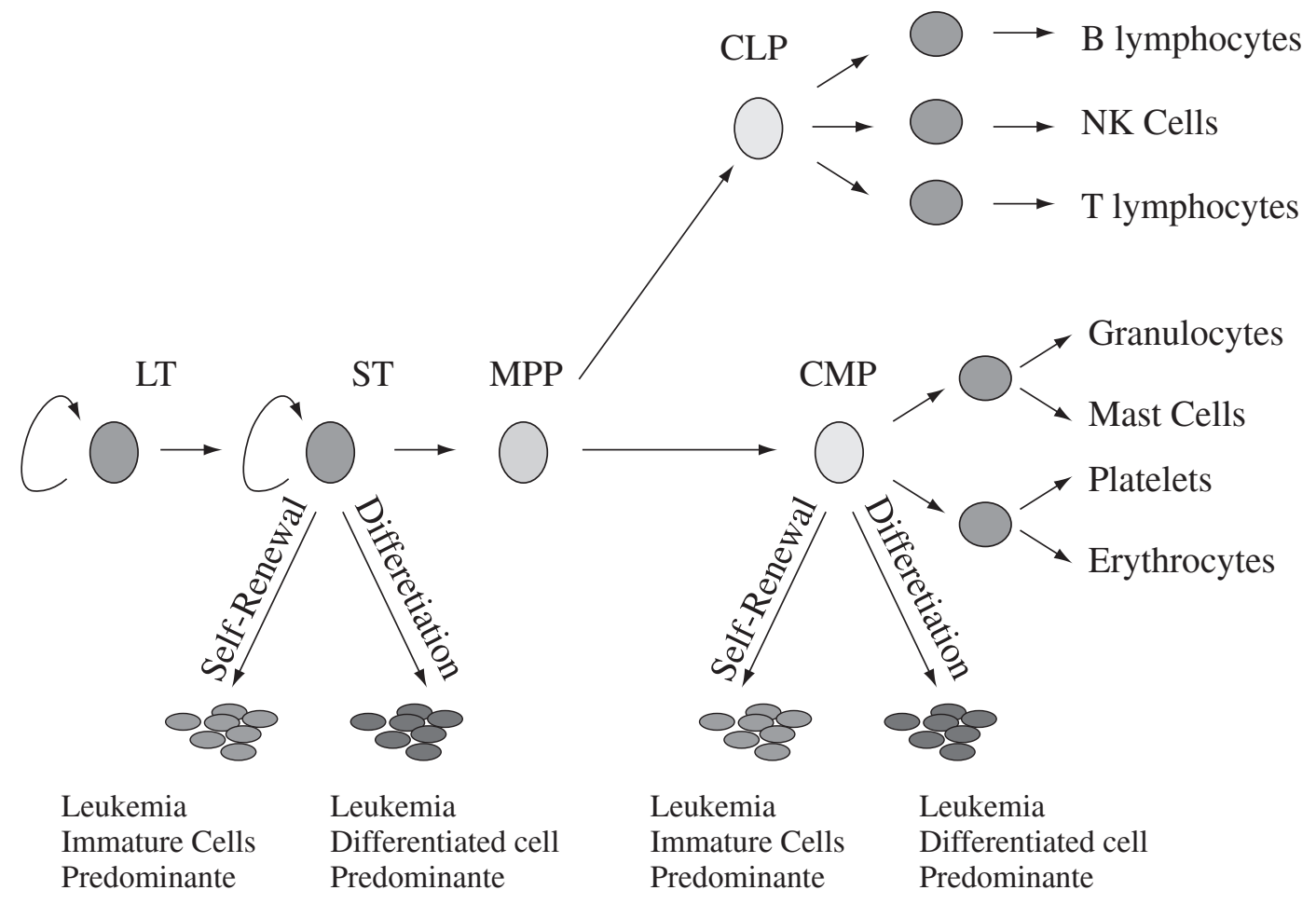

Fig. 1: A hypothetical model for leukemogenesis.

A long-term HSC (LT) is capable of giving rise to short-term (ST) or transient amplifying stem cells. STs further differentiate to multipotent progenitors (MPP), which are then capable of giving rise to the committed lymphoid (CLP) and committed myeloid (CMP) progenitors. The CLPs and CMPs further differentiate to generate all the different types of hematopoietic cells. Leukemia is initiated when a HSC or a progenitor cell is transformed by sequential genetic mutation to a cancer initiating cell or a cancer stem cell. A cancer stem cell may then undergo self-renewal and generate leukemic cells with a similar genetic profile as the originating cell e.g. acute myeloid leukemia; AML. (Adapted according to Behbod F, Rosen JM. Will cancer stem cells provide new therapeutic targets? Carcinogenesis 2004; 26:703-711.) 
As few as one hundred $\mathrm{CD} 44^{+} \mathrm{CD} 24^{-/ \text {low }}$ cells were able to form tumors in mice, whereas cells with alternate surface phenotypes failed to form tumors, even after the transplantation of a million cells. It appears that the microenvironment, especially breast stromal component may play an important role in carcinogenesis (1).

\section{Signal transduction pathways important in breast cancer}

Several signaling pathways are implicated in regulating mammary stem cells. Examples of these pathways are Wnt, Notch, Hedgehog and TGF- $\beta$. Dysregulation of these signaling pathways in the mammary gland has been demonstrated to induce mammary tumors in genetically engineered mice (25). The Wnt gene family encodes growth factors and appears to be hormonally regulated. These two findings together suggest that regulated expression of Wnt gene products play a role in the normal expansion or diffe- rentiation of the mammary epithelium. The alteration of Wnt signaling pathway may cause expansion of stem cells and consequently predispose mammary cells to tumors. Aberrant Notch regulation results in the expansion of specific mammary progenitors, but how the induction of tumorigenesis is still mystery.

\section{Stem/progenitor cells and breast cancer heterogeneity}

Based on variation in gene expression patterns breast carcinoma has been divided into at least five different subtypes with different clinical outcomes (basal like, ERBB2+, normal breast-like, luminal subtype A and luminal subtype B) (43). Based on hypothetical models the heterogeneity in breast cancer reflects the underlying mechanisms involved in cancer initiation in stem/progenitor cells. A cancer-initiating cell or cancer stem cell may self-renew symmetrically giving rise to cancer cells with similar expression profiles

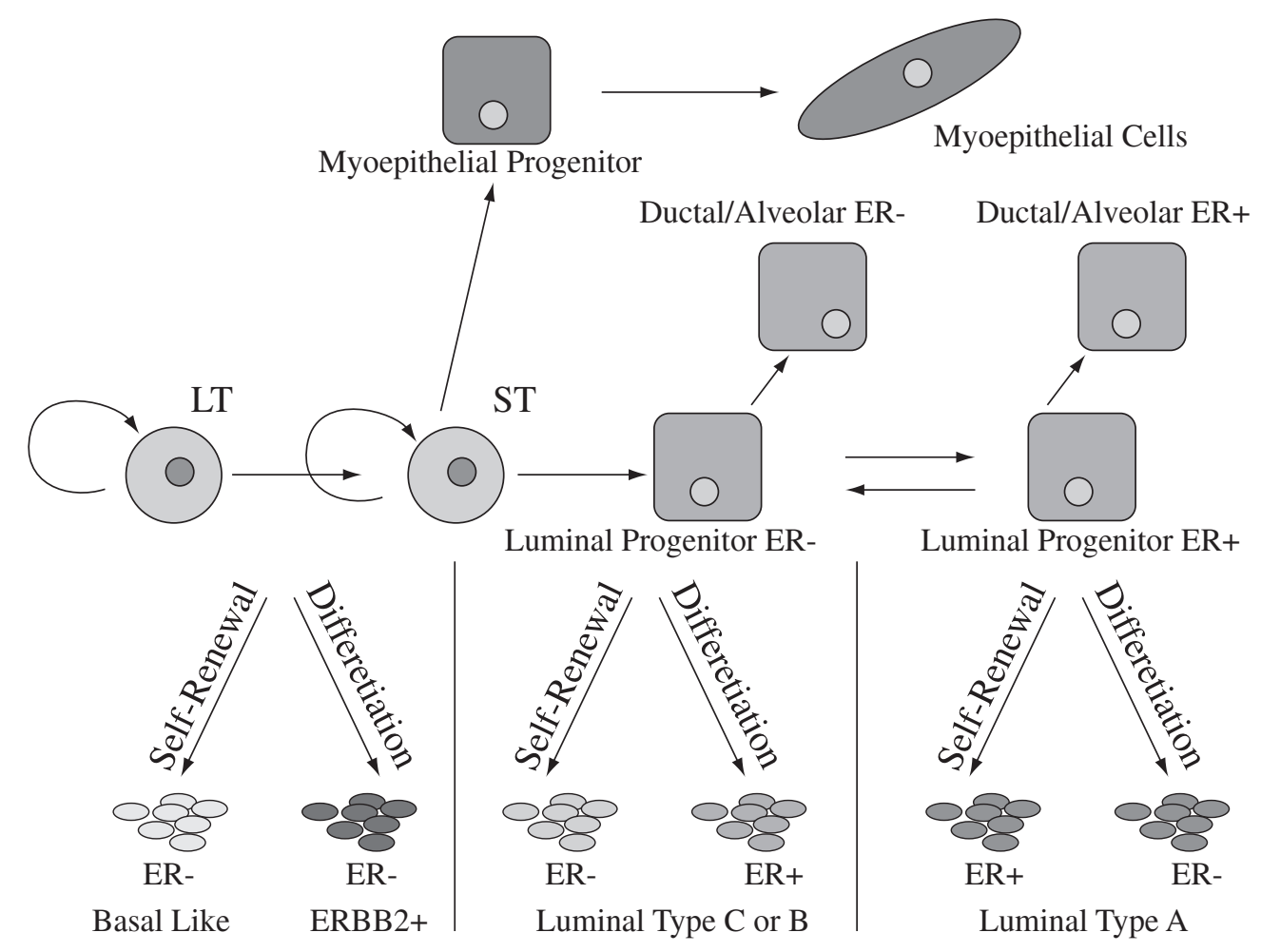

Fig. 2: A hypothetical model of breast cancer development.

Breast cancer is initiated when a progenitor cell acquires sequential mutations resulting in transformation. The cabcer initiating cell or cabccer stem cell may self-renew symmetrically giving rise to cancer cells with similar expression profiles or it may differentiate asymmetrically and generate cancer cells with different genetic profiles, for exemple, an ER cancer stem cell may self-renew giving rise to a population of cancer cells that are ER or it may follow a differentiation path and generate a population of cancer cells that express ER. The tumor bnges resulting in ehavior and response to therapy may be different and dictated by the tumor initiating cells and the type of transforming event. It is conceivable that a normal differentiated cell may undergo genetic or epigenetic changes resulting in transformation, possibly dedifferentiation and self-rennewall. (Adapted according to to Behbod F, Rosen JM. Will cancer stem cells provide new therapeutic targets? Carcinogenesis 2004; 26:703-711.) 
or it may differentiate asymmetrically and generate cancer cells with different genetic profiles. For example an ERcancer stem cell may self-renew giving rise to a population of cancer cells that are ER- or they may follow a differentiation pathway and generate a population of cancer cells that express ER (12). Obviously, these findings could help to explain the response to therapy, but more importantly the clinical outcome.

\section{Brain cancer stem cells}

In recent years, well-established methods for identification, isolation and characterization of brain stem cells have been developed. Studies demonstrate the cancer stem cell concept and furthermore highlight the similarities between somatic stem cells and cancer stem cells in the central nervous system.

Isolated cells from either embryonic tissue or the adult hippocampus, subventricular zone or olfactory bulbs are undifferentiated multipotent neural stem cells which are capable of self-renewal. As first observed in 1992, CNS cells grown on nonadherent surfaces give rise to balls of cells (neurospheres) that have the capacity for self-renewal and can generate all of the principal cell types of the brain (i.e., neurons, astrocytes, and oligodendrocytes) (33, 34). Neurosphere assay is one of the methods used to get more specific details about neural stem cells, such as the potential of self-renewal, expression of specific surface antigens (e.g. CD133) and production of proteins (e.g. nestin).

Progress of knowledge had led to identification of putative cancer stem cells in brain cancer. As known, brain tumors are typically represented by a heterogeneous cell population, containing both undifferentiated and partially differentiated cells. Drik et al. generated neurospheres from 14 solid primary pediatric brain tumors, including medulloblastoma, pilocytic astroytoma, glioblastoma and anaplastic ependymoma (41). Furthermore, brain tumor neurospheres displayed the potential of self-renewal, as well as the ability to give rise to differentiated tumor cells similar to cells of the original tumor. With time there are more and more brain tumor stem cells markers identified for example CD 133, Sox2, Musashi, Bmi-1. The CD 133 surface marker has been used to enrich stem cells from brain tissue material. Singh et al transplanted brain tumor stem cells into nude mice and observed that CD133 positive cancer cells form tumors $(40,41,42)$. It was also demonstrated that brain cancer stem cells drive tumor growth in vivo. In serial transplantation studies only one hundred CD133+ cells were able to initiate and sustain tumor growth in nude mice (41). The study was based on the persistence of a small subpopulation of cancer stem-like cells. Having isolated a putative stem cell population from $\mathrm{C} 6$ glioma cell line, they observed that the C6 glioma subpopulation cells displayed a higher potential for tumor formation and invasiveness compared with non-subpopulation group, although their thesis was statistically insignificant.

\section{Signal transduction pathways important in brain cancer}

If neural stem cells are the source for the initiation of brain cancer, their progression towards a tumorigenic state may be achieved through the operation of abnormal "developmental" programs. There are several described genetic pathways in neural stem-cell regulation and gliomagenesis. Specifically, dysregulation of the sonic hedgehog ( Shh) developmental signaling pathway plays a role for tumor initiation as well as tumor maintenance. The EGF signaling pathway is also important in gliomagenesis and neural stem-cell regulation. Amplification of the EGFR gene is associated with the formation of glioblastomas, and activation of EGFR promotes the growth of both astrocyte precursors $(23,24)$ and neural stem cells $(22)$. PTEN, a tumor-suppressor gene often affected in high-grade gliomas, regulates the proliferation of neural stem cells. PTEN may also play a part in neural stem-cell motility and could therefore lead to the extensive infiltration seen in gliomas (28).

\section{Conclusions and future prospects}

Cancer is increasingly being viewed as a stem cell disease. It has been suggested that tumors contain a small population of putative cancer stem cells with unique selfrenewal and survival mechanisms (12, 21, 32, 38). Despite recent advances in cancer stem cell studies, the knowledge is still limited. Further characterization of cancer stem cells might contribute to the development of more effective treatment strategies. It would be beneficial to investigate, how existing chemotherapeutic methods affect the evolution of stem cells during conventional treatment regiments, for example by comparing the sensitivity of normal stem cells with malignant ones, looking at the mechanisms by which drug resistance may arise. It may not even be necessary to eliminate the cancer stem cells in order to control the cancer, instead it may be sufficient to suppress their growth or redirect the abnormal differentiation towards a more normal phenotype (17).

There is significant evidence that carcinogenesis in the mammary gland, and in other organs are related to deregulation of signaling pathways, like Hedghog, Notch and Wnt. Therefore, it is imperative to design new strategies based upon a better understanding of the signaling pathways that control aspects of self-renewal and survival in cancer stem cells in order to identify novel therapeutic targets in these cells. Furthermore, studies indicate that cancer stem cells are associated with microenvironment niche. These studies could bring forward the prophylactic approach to therapy and the possible prevention.

\section{Acknowledgement}

This work has been supported by Research project MZO 00179906 and MSM 0021620820 from the Ministry of Education Youth and Sport of the Czech Republic. 


\section{References}

1. Al-Hajj M, Wicha MS, Benito-Hernandez A, Morrison SJ, Clarke MF Prospective identification of tumorigenic breast cancer cells. Proc Natl Acad Sci USA 2003;100:3983-3988.

2. Ayton PM, Cleary ML. Molecular mechanisms of leukemogenesis mediated by MLL fusion proteins. Oncogene 2001;20:5695-5707.

3. Ayton PM, Cleary ML. Transformation of myeloid progenitors by MLL oncoproteins is dependet on Hoxa7 and Hoxa9. Genes Dev 2003;17:2298-2307.

4. Behbod F, Rosen JM. Will cancer stem cells provide new therapeutic targets? Carcinogenesis 2004;26:703-711

5. Bonnet D, Dick JE. Human acute myeloid leukemia is organized as a hierarch that originates from a primitive hematopoietic cell. Nature Med. 1997;730-737.

6. Costello T, Mallet F, Gaugler B, Sainty D, Arnoulet C, Gastaut JA, Olive D. Human acute myeloid leukemia CD34+/CD38- progenitor cells have decreased sensitivity to chemotherapy and Fas-induced apoptosis, reduced immunogenicity, and impaired dendritic cell transformation capacities. Cancer Res 2000;60: 4403-4411.

7. Cozzio A, Passegue E, Ayton PM, Karsunky H, Cleary ML, Wiessman IL Similar MLL-Asociated leukemias arising from self-renewing stem cells and short-lived myeloid progenitors. Genes Dev 2003;17:3029-3035.

8. DeOme KB, Faulkin LJ, Bern HA, Blair PB. Development of mammary tumor from hyperplastic alveolar nodules transplanted into gland-free mammary fat pads of female C3H mice. Cancer Res 1959;19:515-520.

9. Dhom G. The cancer cell and the connective tissue. A historical retrospect. Pathologe 1994;15:271-278.

10. Domen J, Gandy KL, Weissmann IL. Systemic overexpression of BCL2 in the hematopoietic system protects transgenic mice rom the consequens of irradiation. Blood 1998;91:2272-2282.

11. Domen J, Weissman IL. Hematopoietic stem cells need two signals to prevent apoptosos; BCL-2 can provide one of these, Kit/c-Kit signaling the otherJ Exp Med 2000;192:1707-1718

12. Dontu G, El-Ashry D, Wicha MS. Breast cancer, stem/progenitor cells and th estrogen receptor. Trends Endocrinol Metab 2004;15:193-187.

13. Filip S, English D, Mokrý J. Issues in stem cell plasticity. J Cell Mol Med 2004;8:572-577.

14. Filip S, Mokrý J, English D. Stem cell plasticity and carcinogenesis. Neoplasma 2006;53:87-91.

15. Filip S. The phenomenon of stem cell plasticity: biological or physiological problems? Stem Cell Dev 2006;15:753

16. Guan Y, Gerhard B, Hogge DE. Detection, isolation, and stimulation of quiescen primitive leukemic progenitor cells from patients with acute myeloid leukemia (AML). Blood 2003:101:3142-3149.

17. Gudjonsson T, Magnusson MK. Stem cell biology and the cellular pathways of carcinogenesis. APMIS 2005;113:922-929.

18. Jordan CT, Guzman ML, Noble M. Cancer stem cells. N Engl J Med 2006; 355 : $1253-1261$.

19. Kopper L, Hajdu M. Tumor stem cells. Pathol Oncol Res 2004;10:69-73.

20. Kordon EC, Smith GH. An entire functional mammary gland may comprise the progeny from single cell. Development 1998;125:1921-1930.

21. Lessard J, Sauvageau G. Bmi-1 determines the proliferative capacity of normal and leukemic stem cells. Nature 2003:423:255-260.

22. Lewis MT, Ross S, Strickland PA, Sugnet CW, Jimenez E, Hui C, Daniel CW. The Gli2 transcription factor is required for normal mouse mammary gland development. Dev Biol 2001;238:133-144.

23. Lewis MT, Ross S, Strickland PA, Sugnet CW, Jimenez E, Scott PM, Daniel CW. Defects in mouse mammary gland development caused by conditional haploinsuffiency of Patched-1. Developement 1999;126:5181-5193.
24. Lewis MT. Hedgehog signaling in mouse mammary gland development and neoplasia. J Mammary Gland Biol Neoplasia 2001;6:53-66.

25. Liu BY, McDermott SP, Khwaia SS, Alexander CM. The transforming activity of Wnt effectors correlates with their ability to induce the accumulation of mammary progenitor cells. Proc Natl Acad Sci USA 2004;101:4158-4163.

26. Marx J. Cancer research; Mutant stem cells may seed cancer. Science 2003; 301:1308-1310.

27. Moore MA, Williams N, Metcalf D. In vitro colony formation by normal and leukemic human hematopoietic cells: interraction between colony-forming and colony-stimulating cells. J Natl Cancer Inst. 1973;50:591-602.

28. Morshead CM, Reynolds BA, Craig CG, Magburney MW, Staines WA, Morassutti D, Weiss S, VanDerKooy D. Neural stem cells in the adult mammalian forebrain: a relatively quiescent subpopulation of subependymal cells. Neuron 1994 13:1071-1082

29. Park IK, Qian D, Kiel M, Becker MW, Pihalja M, Weissman IL, Morrison SJ, Clarke MF. Bmi-1 is required for maintenance of adult self-renewing haematopoietic stem cells. Nature 2003:423:255-260.

30. Pierce GB, Johnson LD. Differentiation and cancer. In Vitro 1971;7:140-145.

31. Quesenberry PJ, Colvin GA, Adebi M, Dooner G, Dooner M, Aliotta J, Keaney P, Luo L, Demers D, Peterson A, Foster B, Greer D. The stem cell continuum. Ann NY Acad Sci 2005;1044:228-235.

32. Reya T, Morrison SJ, Clarke MF, Weissman IL. Stem cells, cancer and cancer stem cells. Nature 2001;414:105-111.

33. Reynolds BA, Tetzlaff W, Weiss S. A multipotent EGF-responsive striatal embryonic progenitor cell produces neurons and astrocytes. J Neurosci 1992;12: 4565-4574

34. Reynolds BA, Weiss S. Generation of neurons and astrocytes from isolated cell of the adult mammalian central nervous systém. Science 1992;255:1707-1710.

35. Robinson WA, Kurnick JE, Pike BL. Colony growth of human leukemic peripheral blood cells in vitro. Blood. 1971:38:500-508.

36. Schreiner S, Birke M, Garcia-Cuellar MP, Zilles O, Greil J, Slany RK. MLL-ENL causes a reversible and myc-dependent block of myelomonocyte cell diffrentiation. Cancer Res 2001;61:6480-6486.

37. Sell S, Pierce GB. Maturation arest of stem cell differentiation is a common pathway for the cellular origin of teratocarcinomas and epithelial cancers. Lab Invest 1994;70:6-22.

38. Sell S. Stem cell origin of cancer and differentiation therapy. Crit Rev Onco Hematol. 2004:51:1-28.

39. Simonovitch L, McCulloch EA, Till JE. The distribution of colony-forming cells among spleen colonies. J Cell Physiol. 1963;62:327-336.

40. Singh SK, Clarke LD, Hide T, Dirks PB. Cancer stem cells in nervous system tumors. Oncogene 2004;23:7267-7273.

41. Singh SK, Clarke LD, Terasaki M, Bonn VE, Hawkins C, Squire JA, Dirks PB. Indentification of cancer stem cell in human brain tumors. Cancer Res 2003; 63:5821-5828

42. Singh SK, Hawkins C, Clarke ID, Squire JA, Bayani J, Hide T, Henkelman RM, Cusimano MD, Dirks PB. Identification of human brain tumour initiating cells. Nature 2004:432:396-400

43. Sorlie T, Tibshirani R, Parker J et al. Repeated observation of breast tumor subtypes in independent gene expression data sets. Proc Natl Acad Sci USA 2003; 100:8418-8423.

44. Spradling A, Drummond-Barbosa D, Kai T. Stem cells find their niche. Nature 2001;414:98-104.

45. Taiple J, Beachy PA. The Hedgehog and Wnt signaling pathways in cancer Nature 2001;411:349-354.

46. Trosko JE, Chang CC. Stem cell theory of carcinogenesis. Toxicol Lett. 1989; 49:283.

47. Welm BE, Tepera SB, Venezia T, Graubert TA, Rosen JM, Goodell MA. Sca-1 (pos) cells in the mouse mammary gland represent an enriched progenitor cell population. Dev Biol 2002;245:42-56.

Submitted March 2008. Accepted August 2008.

\section{Corresponding author:}

Zuzana Mačingová, M.D., Department of Oncology and Radiotherapy, University Hospital, Sokolská 581, 50005 Hradec Králové, Czech Republic; e-mail: macinzuz@fnhk.cz 